\title{
Effects of polymerization degree on recovery behavior of PVA/PVP hydrogels as potential articular cartilage prosthesis after fatigue test
}

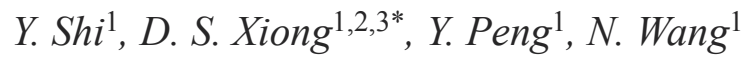 \\ ${ }^{1}$ School of Materials Science and Engineering, Nanjing University of Science and Technology, 210094 Nanjing, Jiangsu, \\ PR China \\ ${ }^{2}$ Jiangsu Key Laboratory of Advanced Micro/nano Materials and Technology, 210094 Nanjing, Jiangsu, PR China \\ ${ }^{3}$ Synergetic Research Center on Advanced Materials (SRCAM), 210094 Nanjing, Jiangsu, PR China
}

Received 13 April 2015; accepted in revised form 19 September 2015

\begin{abstract}
Poly (vinyl alcohol)/poly (vinyl pyrrolidone) (PVA/PVP) hydrogels with various polymerization degrees of PVA were synthesized by a repeated freezing-thawing method. The influence of polymerization degree on microstructure, water content, friction coefficient, compressive fatigue and recovery properties of PVA/PVP hydrogels were investigated. The results showed that higher polymerization degree resulted in larger compressive modulus and lower friction coefficient. The fatigue behaviors of PVA/PVP hydrogels were evaluated under sinusoidal compressive loading from 200 to $800 \mathrm{~N}$ at $5 \mathrm{~Hz}$ for up to 50000 cycles. The unconfined uniaxial compressive tests of PVA/PVP hydrogels were performed before and after fatigue test. During the fatigue test, the height of the hydrogel rapidly decreased at first and gradually became stable with loading cycles. The compressive tangent modulus measured $0 \mathrm{~h}$ after fatigue was significantly larger than the values obtained before test, and then the modulus recovered to its original level for $48 \mathrm{~h}$ after test. However, the geometry of hydrogels could not return to the original level due to the creep effects. PVA/PVP hydrogels prepared with lower polymerization degree showed better recovery capability than that prepared with high polymerization degree.
\end{abstract}

Keywords: mechanical properties, PVA/PVP hydrogel, polymerization degree, fatigue, recovery

\section{Introduction}

Articular cartilage, which covers the end of the long bones in the body, functions as a nearly frictionless bearing, and withstands high mechanical loads and uniformly transfers the loads on underlying bone to prevent high stress concentrations. Because of the avascular and aneural nature of articular cartilage it endows itself with poor intrinsic healing capacity for self-repair [1]. Once the articular cartilage is damaged, osteoarthritis gradually develops and finally the joint loses its function [2]. The surgical techniques, including microfracture, autologous chondrocyte implantation (ACI), osteochondral autograft transfer (OAT) etc. [3], have been widely used to ease pain and regenerate tissue function. However, all of these techniques cannot reconstruct natural function to the cartilage. Using an appropriate implant to replace the damaged cartilage is considered as a promising approach for cartilage replacement and has been of great interest to biomaterial scientists for many years [4].

Hydrogels, which are hydrophilic polymers that are swollen in water [5], are widely researched as a potential artificial articular cartilage due to their structural similarity to cartilage [6]. As present, hydrogels have been used in two forms for the pur-

\footnotetext{
${ }^{*}$ Corresponding author, e-mail: xiongds@163.com

(C) BME-PT
} 
pose of articular cartilage repair - as permanent implants to replace damaged cartilage, or as cell carrier materials to encourage tissue regeneration [7]. Hydrogels that consist of naturally derived materials, like hyaluronan [8], collagen [9], chitosan [10], alginate [11], etc, are often used as cell-seeded tissue engineering scaffold. Though these hydrogels have been successful in mimicking the morphological and biochemical appearance of hyaline cartilage, but the lack of mechanical integrity makes them inappropriate as load-bearing implants [7]. Synthetic polymers, including PVA [12, 13], poly(ethylene glycol) (PEG) [14], poly( $N$-isoproylacrylamide) (PNIPAAm) $[15,16]$, show mechanical similar to cartilage and allow efficient load transfer [17]. PVA hydrogel has been one of the most widely used polymer as a potential articular cartilage substitute due to its excellent biocompatibility, good load-bearing properties, and can be easily processed and modified $[12,18]$. The swelling behavior $[19,20]$, mechanical properties [21, 22], and biotribological properties [23-25] of PVA hydrogels has been widely characterized by researchers. Tadavarthy et al. [26] found no adverse effects in surrounding tissue after implanting PVA gels subcutaneously or intramuscularly into rabbits. Baker et al. [18] summarized that PVA hydrogels could be tailored to 1-17 MPa of tensile strength and 0.0012-0.85 MPa of compressive modulus, which is close to the natural cartilage, by changing polymer concentration and the number of cycles tested.

However, pure PVA hydrogels undergo dissolution when immersed in a solvent for a long period of time. The dissolution mechanism involves the unfolding of the polymer chains of crystals in the presence of water to join the amorphous portion surrounding them [27], which leads to larger pore sizes and hence lower mechanical stiffness. PVP is another hydrogel that has been used for a variety of biomedical applications due to its good biocompatibility and hydrophilic ability properties [28, 29]. The incorporation of PVP into PVA is expected to significantly decrease degradation and stabilize the polymer network and the mechanical properties of pure PVA hydrogels through hydrogen bonding interactions between the carbonyl group on PVP and the hydroxyl group along PVA chain [30]. Leone et al. [31] has demonstrated the biocompatibility of PVA/PVP hydrogel. Thomas et al. [32] indicated that blend hydrogels prepared with $99 \mathrm{wt} \%$ PVA and $1 \mathrm{wt} \%$
PVP had the best combination of network stability and a relatively tight, stable, and crosslinked network. Our previous study showed the consistent results with Thomas' that PVA/PVP hydrogel with $1 \mathrm{wt} \%$ PVP had the best mechanical strength [21]. The term fatigue is used to describe the changes in properties of metallic and non-metallic materials resulting from a repeatedly applied stress or strain. If applied adequate number of times, failure may occur in the materials. During daily activities, the synovial joint is routinely subjected to static as well as dynamic loads. It has been estimated that the average person takes about 2 millions steps per year [33]. Therefore, the articular cartilage on the diarthrodial joint in the lower limbs is exposed to approximately 1 millions load cycles during this time, and each cyclic load is of several times body weight in walking condition $[33,34]$. During exercise such as running, cartilage is also subjected to cyclic loading and the loads are applied at faster rates than that experienced during walking. There have been a number of researches shown that the appropriate dynamic mechanical stimulation on cell-seeded tissue-engineered constructs is beneficial to enhance extracellular matrix (ECM) synthesis, or stimulate proliferation and chondrogenesis of progenitor cells [35-38]. However, fatigue resulting from cyclic load was suggested as a failure mechanism of cartilage [39]. Weightman [40] demonstrated that the fatigue properties of human articular cartilage decreased with age, and fatigue failure could occur in life to such an extent. It was thought that the reduction in mechanical properties of cartilage with age was itself the result of fatigue [40]. Therefore, it is of high importance to study the alternative materials for cartilage replacement from a fatigue point of view attributed to the presence of cyclic stress that the materials would face in vivo after implantation. Vikingsson and coworkers [41, 42] proposed a model to assess mechanical behavior of polycaprolactone (PCL) scaffold and predict their performance 'in vivo' during tissue regeneration, the results showed that the scaffold/gel construct was not affected after up to 100000 compressive dynamic cycles. But this kind of scaffold would degrade gradually after implantation in vivo, followed by the decreasing load-bearing capacity.

When the fatigue properties of cartilage replacements were investigated, the researchers generally used dynamic loading with strain controlled, and the strain 
was limited to $15 \%$, which is considered to be the order of magnitude of physiological deformations suffered by articular cartilage [41]. 15\% strain always corresponds to a very small load for hydrogels, just several $\mathrm{N}$ and even lower for tissue engineered hydrogels. This load is far less than the physiological load that the cartilage is subjected. And $15 \%$ strain is very close to the linear elastic region of hydrogels, so it is expected that the hydrogel would not fail for even a very long term loading cycles. To our knowledge, there are few reports about the recovery behavior of cartilage prosthesis after fatigue test under large load.

In the present study, PVA hydrogels with small amounts of PVP were synthesizes by a repeated freezing-thawing method. The effects of the incorporation of PVP and the polymerization degree of PVA on the material properties of PVA/PVP hydrogels, including microstructure, water content, compressive mechanical and friction properties, were evaluated. In order to explore the compressive fatigue properties of PVA/PVP hydrogels as cartilage prosthesis, the hydrogel samples were subjected to cyclic compressive loading from 200 to $800 \mathrm{~N}$ for up to 50000 cycles at $5 \mathrm{~Hz}$. The changes in height and compressive tangent modulus before and after fatigue tests, and the recovery behavior of PVA/PVP hydrogels with time after fatigue tests were investigated.

\section{Materials and methods}

\subsection{Hydrogel preparation}

PVA, saponified greater than $99 \%$ with a polymerization degree of 1750, 2099 and 2399, and PVP K30 (40000 g. $\mathrm{mol}^{-1}$, Shanghai Jiuyi Chemical Reagent Co., China) were chosen as the raw materials. Both polymers were used without further purification. The required amount of polymer powders were dissolved in deionized water at $90^{\circ} \mathrm{C}$ for $10 \mathrm{~h}$ to prepare $15 \mathrm{wt} \%$ polymer solution, composed of $99 \mathrm{wt} \%$ PVA and $1 \mathrm{wt} \%$ PVP. And then the polymer solution was held at a higher temperature to remove air bubbles. After that the polymer solution was then poured into moulds and subjected to 6 cycles of freezing at $-20^{\circ} \mathrm{C}$ for $18 \mathrm{~h}$ and thawing at room temperature for $6 \mathrm{~h} .15 \mathrm{wt} \%$ pure PVA 1750 hydrogels were prepared as controls.

\subsection{Microstructure}

Scanning electron microscopy (SEM, JEOLJSM$6380 \mathrm{LV}$, Japan) was used to evaluate the morphol- ogy and microstructure of PVA/PVP hydrogels. The samples were dried by vacuum oven at $50^{\circ} \mathrm{C}$ for $60 \mathrm{~h}$ to remove all water, and then they were sputtercoated with a layer of gold for SEM observations. The porosity was investigated by image processing using $\mathrm{VC}++$ software. Briefly, scanning electron micrographs of hydrogels were threshold analysed on the basis of different grayscale. The porosity of specimens was calculated via the ratio of black pixel area to gross. Three SEM pictures at different region for each hydrogel formulation were chosen to calculate the porosity.

\subsection{Differential scanning calorimetry analysis} Differential scanning calorimetry (DSC, MettlerToledo, Switzerland, Model: DSC823e) was performed on 3 independent specimens for each hydrogel formulation to examine crystallinity. Dynamic scans were carried out from 50 to $300^{\circ} \mathrm{C}$ at a heating rate of $10^{\circ} \mathrm{C} / \mathrm{min}$, under $\mathrm{N}_{2}$ atmosphere. The speed of nitrogen gas flow was $30 \mathrm{~mL} / \mathrm{min}$. Before DSC analysis, the samples were dried in vacuum for at least $48 \mathrm{~h}$ at $60^{\circ} \mathrm{C}$. The crystallinity degree was calculated using Equation (1) and the heat of fusion of a perfect crystal of PVA sample $\left(\Delta H_{\mathrm{f}^{*}}=138.6 \mathrm{~J} / \mathrm{g}\right)$ [43]:

Crystallinity $=\frac{\Delta H_{\mathrm{f}}}{\Delta H_{\mathrm{f}^{*}}} \cdot 100$

\subsection{Water content}

For all the hydrogel samples, the water content, $W$, was determined according to Equation (2):

Water content $=\frac{M_{\mathrm{s}}-M_{\mathrm{d}}}{M_{\mathrm{s}}}$

where $M_{\mathrm{s}}$ and $M_{\mathrm{d}}$ represent the equilibrium swollen and completely dried sample, respectively. Five independent samples were tested for each set of hydrogels $(n=5)$.

\subsection{Mechanical characterization}

\subsubsection{Compression tests}

Unconfined compressive properties were carried out in a PBS solution bath at room temperature on Instron 5943 under three different strain rate (100 and $1000 \% \cdot \mathrm{min}^{-1}$ ) up to $65 \%$ strain to investigate the strain rate effect on compression property. The compressive tangent modulus for each hydrogel sample was calculated at $10 \%$ increments from 10 
$60 \%$ strain by the finite difference method according to the stress-strain curves obtained, and it was expressed with the Equation (3) [44]:

$E_{\varepsilon}=\frac{\sigma_{\varepsilon+\Delta \varepsilon}-\sigma_{\varepsilon-\Delta \varepsilon}}{2 \Delta \varepsilon}$

where $E_{\varepsilon}$ is the compressive tangent modulus of the hydrogel at the compression strain ratio value of $\varepsilon$. The value of $\Delta \varepsilon$, the variable of strain value, is $2 \%$. The sample size was approximately $12 \mathrm{~mm}$ in diameter and $4 \mathrm{~mm}$ in height. Three independent samples were tested for each set of hydrogels $(n=3)$.

\subsubsection{Indentation creep test}

The indentation creep tests were conducted in an UMT-IImulti-functional micro-friction test machine in PBS solution at room temperature. The hydrogel samples were manufactured into cylindrical disks with $20 \mathrm{~mm}$ in diameter and $4 \mathrm{~mm}$ in thickness. A stainless steel ball (Stellite 3) was used as the indenter, and its diameter was $7.9375 \mathrm{~mm}$. The samples were placed between the compression plate and the spherical indenter under compression to $10 \mathrm{~N}$, resulting in a nominal contact stress of about $0.6 \mathrm{MPa}$. And then this load was kept constant for $3600 \mathrm{~s}$. The displacement and time were recorded. Three indentations at different region were indented for each hydrogel formulation.

\subsubsection{Viscoelastic measurements}

Dynamic shear tests were conducted on a controlled strain dynamic mechanical thermal analysis instrument (DMTA Q800, USA), and the samples were subjected to a sinusoidal shear strain at room temperature with frequencies ranging from 0.1 to $10 \mathrm{~Hz}$. The strain amplitude of $3 \%$ was used, which was within the range of linear viscoelastic region. The flat rectangular samples with $25 \mathrm{~mm}$ parallel plates were used. The dynamic viscoelastic functions such as $G^{\prime}$ (storage modulus) and $G^{\prime \prime}$ (loss modulus) and were measured as function of frequency.

\subsubsection{Compressive fatigue tests}

Compressive fatigue tests were performed on Instron Model 8801 test machine. The samples were placed under an impermeable compression plate, and subjected to dynamic compressive load from 200 to $800 \mathrm{~N}$ at $5 \mathrm{~Hz}$, using a sinusoidal waveform, for up to 50000 cycles in PBS at room temperature. The tests were run with a preload of approximately $500 \mathrm{~N}$.
The displacement was simultaneously recorded with 20 data point per compression cycle during the whole fatigue test, which presented the relative position of the press head, and reflected the deformation of the hydrogel in the process of fatigue test. The samples were immersed back in fresh PBS at room temperature for up to 14 days, with height and mass measured every day. Compression tests were carried out on an electronic universal material testing machine (CSS-44300, China) on each hydrogel sample before fatigue test, and $0 \mathrm{~h}$ (immediately after the test), 24, $48 \mathrm{~h}$ and 14 days after fatigue test under a strain rate of $100 \% \cdot \mathrm{min}^{-1}$. The compressive tangent modulus was calculated at 10, 20, and 30\% strain. The hydrogel samples were manufactured into rectangle with the size of $21 \mathrm{~mm} \times 15 \mathrm{~mm} \times 9 \mathrm{~mm}$. As the hydrogel is slippery due to its very smooth surface, the hydrogels were packed with ultra-high molecular weight polyethylene (UHMWPE) fiber cloth, and the cloth would limit the lateral expansion of hydrogels and raised the load-bearing capacity. Three independent samples were tested for each hydrogel formulation $(n=3)$.

\subsection{Friction tests}

The friction property was evaluated by sliding against CoCrMo ball with a diameter of $8 \mathrm{~mm}$ on a ball-onplate reciprocating sliding tribometer (CETR-UMT2, USA). Tests were conducted at room temperature lubricated by PBS solution. The applied normal load, reciprocating sliding frequency and stroke length were kept constant at $5 \mathrm{~N}, 1 \mathrm{~Hz}$ and $8 \mathrm{~mm}$, respectively. Test duration was typically $30 \mathrm{~min}$, and the friction coefficient evolution was continuously monitored during the tests. Each of test was repeated three times $(n=3)$.

\subsection{Statistical analysis}

All values were reported as means of standard deviation. Statistical analysis was performed using a oneway analysis of variance (ANOVA). Comparison between the two means was made by the Tukey test with statistical significance evaluated at $p<0.05$.

\section{Results}

\subsection{Microstructure}

Investigation of PVA hydrogel microstructure indicates that the hydrogel shows an internal threedimensional network structure with lots of micropores on the surface (Figure 1a), which is similar to 
SEM micrographs of the natural cartilage dried under the same condition [45]. As demonstrated by researchers, phase separation into a PVA-rich phase and a water-rich phase is the crucial and unique mechanism that occurs in the process of freezethawed PVA hydrogel gelation [46]. During freezing process, water froze, while the polymers were expelled to form regions of concentrated polymer. The polymer chains in polymer-rich regions were pushed into close contact with each other, facilitating the formation of hydrogen bonding and crystallite. During the thawing process, the crystals of frozen water, which performed as a pore-forming agent, left pores filled with water. These processes repeated with each cycle, microstructural remodeling of the polymer phase occurred, decreasing the distance between the polymer chains further and promoting crosslinking; water crystals grew and even met the facets of other crystals during freezing, and then interconnected pores arose within the

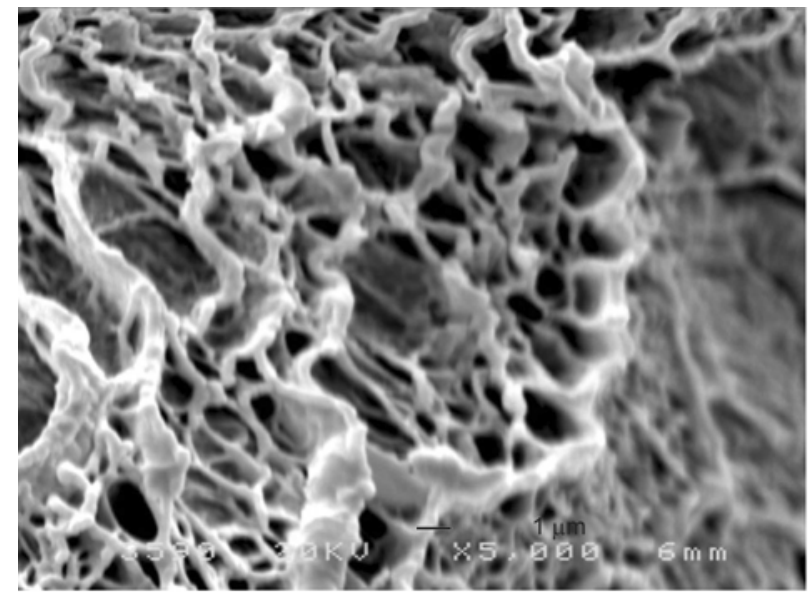

a)

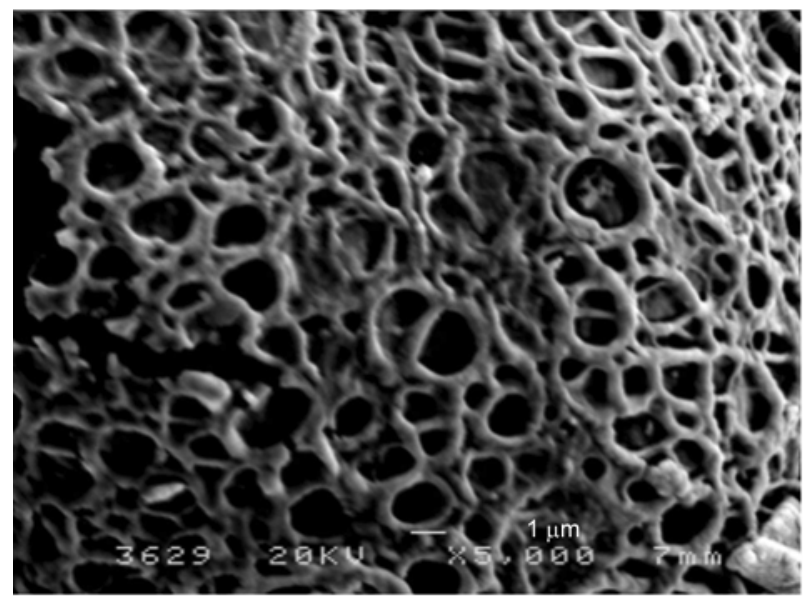

c) hydrogel after thawing. Finally a three-dimensional and porous and interconnected hydrogel structure was created. When blended with PVP, the internal structure of PVA/PVP hydrogels becomes denser and less porosity (Figure 1b) due to the interpolymer complexation between PVA and PVP. With the increase of polymerization degree of PVA, the network becomes denser, and the porosity decreases, as shown in Table 1. For long PVA molecular chains, it is believed to provide more physical crosslinking points, encouraging the formation of more hydrogen bonding and hence a denser network structure.

\subsection{Crystallinity}

The degrees of crystallinity of PVA hydrogels were listed in Table 1. The incorporation of PVP increased the crystallinity of PVA hydrogels, while the crystallinity decreased with the increase of PVA polymerization degree. That is because the higher polymerization degree results in greater crosslinking,

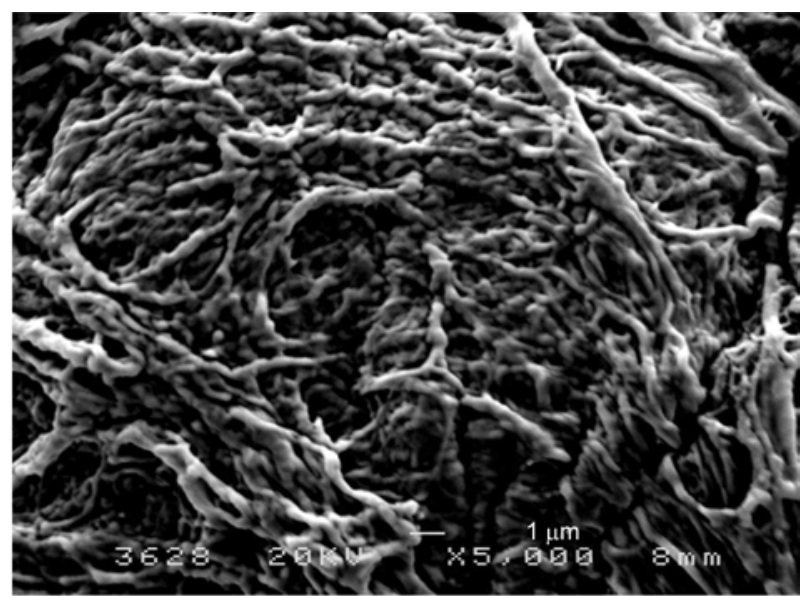

b)

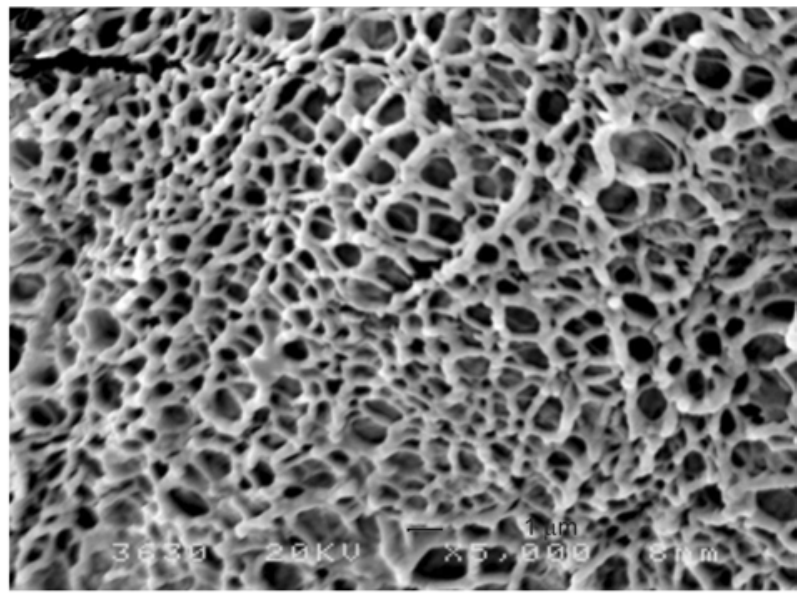

d)

Figure 1. Micrographs of pure PVA hydrogel and PVA/PVP hydrogels prepared with various polymerization degree of PVA. (a) Pure PVA1750 hydrogel, (b) PVA1750/PVP hydrogel, (c) PVA2099/PVP hydrogel, (d) PVA2399/PVP hydrogel. 
Table 1. Porosity, crystallinity, water content, and friction coefficient of PVA/PVP hydrogels

\begin{tabular}{|l|c|c|c|c|}
\hline \multicolumn{1}{|c|}{ Sample } & $\begin{array}{c}\text { Porosity } \\
{[\text { \%] }}\end{array}$ & $\begin{array}{c}\text { Crystallinity } \\
{[\%]}\end{array}$ & $\begin{array}{c}\text { Water content } \\
{[\%]}\end{array}$ & Friction coefficient \\
\hline Pure PVA1750 & $79.39 \pm 5.52$ & $35.1 \pm 1.2$ & $81.3 \pm 1.5$ & 0.261 \\
\hline PVA1750/PVP & $72.69 \pm 4.63$ & $36.2 \pm 1.8$ & $84.6 \pm 1.2$ & 0.193 \\
\hline PVA2099/PVP & $65.32 \pm 5.21$ & $28.1 \pm 1.7$ & $82.2 \pm 1.0$ & 0.182 \\
\hline PVA2399/PVP & $56.76 \pm 3.68$ & $21.5 \pm 2.1$ & $80.8 \pm 0.7$ & 0.139 \\
\hline
\end{tabular}

which may break the symmetry and regularity of the polymer chains, and the resulting denser structure limits the activities of polymer chains, and then the crystallinity of the hydrogel decreases significantly $(p<0.05)$.

\subsection{Water content}

The values of water content of different PVA hydrogels are summarized in Table 1. It is shown that the addition of PVP into PVA hydrogel resulted in the increase of water content of the pure hydrogel, because the amide group on PVP showed stronger hydrophilicity than the hydroxyl group on PVA [21]. The polymerization degree of PVA showed a significant influence on water content of hydrogels. The water content decreased significantly $(p<0.05)$ with the increasing polymerization degree attributed to the denser network structure, which left less space for water molecules to inhabit.

\subsection{Compressive mechanical properties}

\subsubsection{Unconfined uniaxial compression tests}

As shown in Figure 2, the unconfined uniaxial compressive properties of PVA/PVP hydrogels were found to be significantly influenced by strain level, which demonstrated the typically nonlinear and viscoelastic behavior. The addition of PVP enhanced the mechanical property of pure PVA hydrogel. The tangent modulus significantly increased with the increasing polymerization degree of PVA. But the differences of the tangent modulus value between PVA1750/PVP and PVA2099/PVP hydrogels were not significant when the strain level was below $30 \%$. The compressive tangent modulus increased about tenfold from 30 to $60 \%$ strain. At low strain level, the hydrogels exhibited small tangent modulus and were prone to deformation, so that the pressure presented on the materials could be uniformly distributed by the enlarged contact area. While at high strain level, the larger tangent modulus of the materials led to the ability to withstand larger stress and resist the deformation. These are consistent with the important functions of natural cartilage to support and distribute large mechanical loads [47]. The compressive tangent modulus shows strong dependence on strain rate (Figure 2b). For each hydrogel formulation, the high strain rate resulted in a significant increase of the tangent modulus at the same strain level. It is because the interstitial water had no time to migrate out from the pores under large strain rate, resulting in higher instantaneous pressurization to sustain higher stress, and hence the compressive tangent modulus at the same strain level increased.
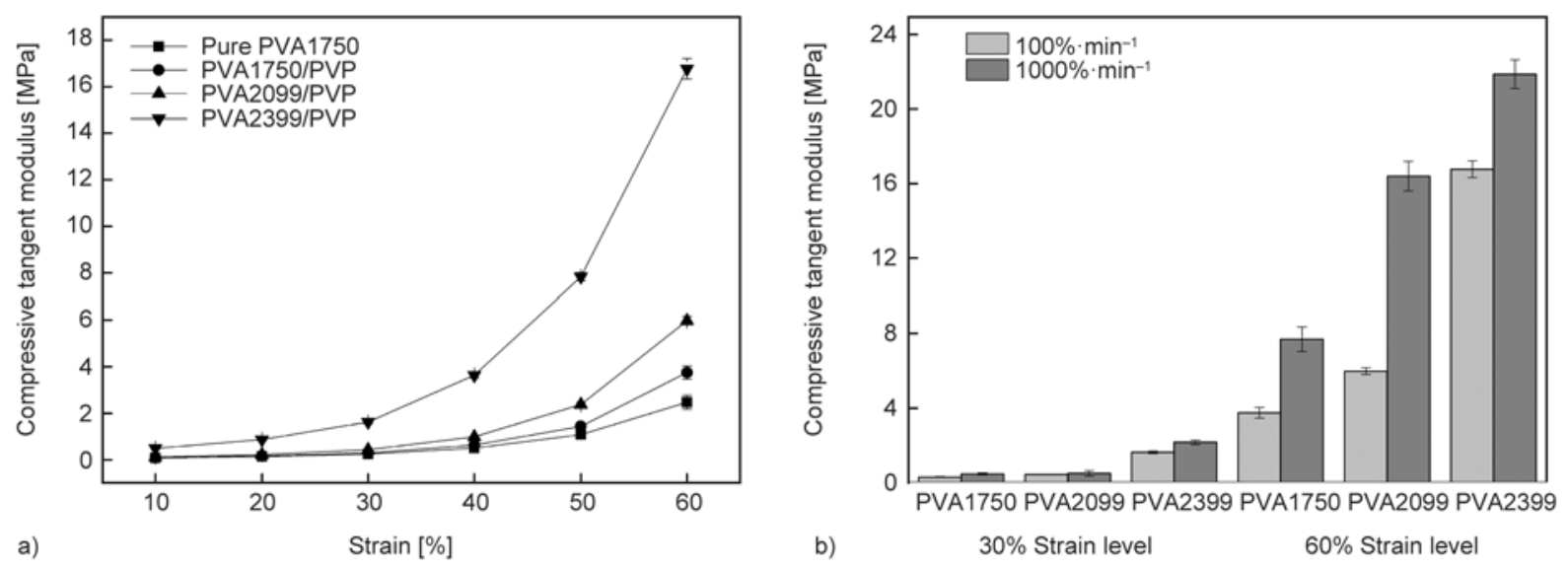

Figure 2. Effects of (a) strain magnitude $\left(100 \% \cdot \mathrm{min}^{-1}\right)$ and (b) the strain rate on the compressive tangent modulus of various hydrogel formulations 


\subsubsection{Indentation creep test}

Figure 3 demonstrates the indentation creep properties of various PVA/PVP hydrogel formulations. It was found that under constant compressive stress, the deformation rate of all hydrogel samples rapidly increased at first, and then became slow with time, and it may infer from the deformation-time curves that the deformation would reach equilibrium state eventually. The incorporate of PVP and higher polymerization degree of PVA resulted in less deformation of the hydrogels due to the resulting increased compressive modulus $(p<0.05)$. In the end of the test, the deformation of pure PVA1750, PVA1750/PVP, PVA2099/PVP and PVA2399/PVP hydrogel samples were $0.4897 \pm 0.021,0.435 \pm 0.016,0.403 \pm 0.011$ and $0.358 \pm 0.012 \mathrm{~mm}$, respectively.

\subsection{Viscoelastic behavior}

Figure 4 displays the shear storage modulus $\left(G^{\prime}\right)$ and loss modulus $\left(G^{\prime \prime}\right)$ as a function of frequency for each hydrogel tested. It can be seen that for all hydrogel formulations, $G^{\prime}$ was always much higher than

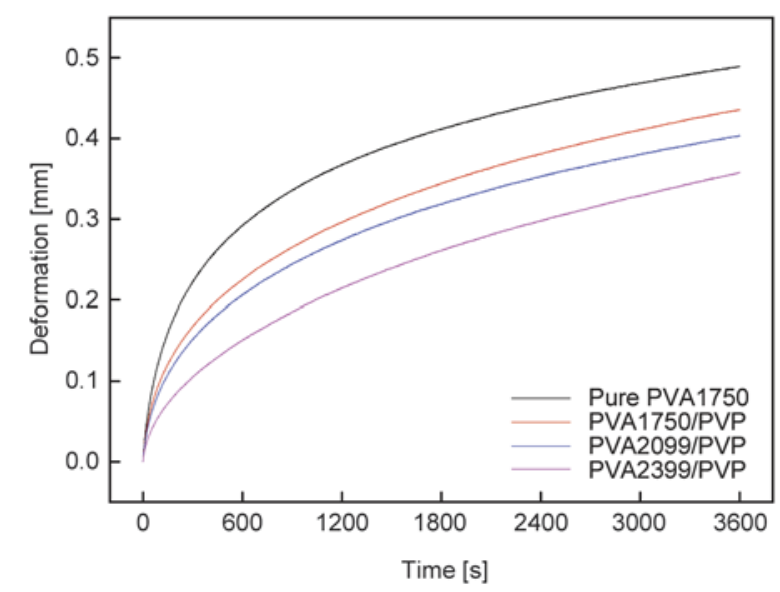

Figure 3. Effect of polymerization degree of PVA on indentation creep characteristics of PVA/PVP hydrogels

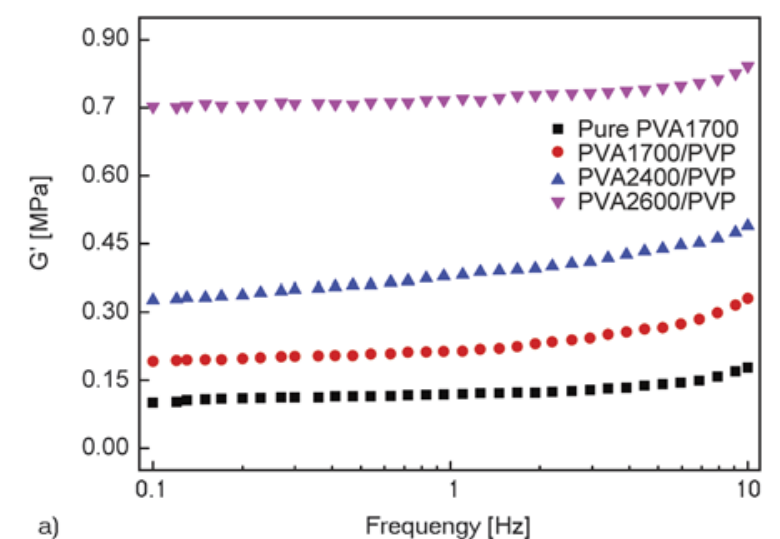

$G^{\prime \prime}$ over the whole frequency range, which was a characteristic feature of a 'strong' hydrogel [48]. $G^{\prime}$ values of all hydrogel samples increased slowly with the increasing test frequency, implying the hydrogels maintain a strong network structure [49]. The similar tendency of $G^{\prime \prime}$ varying with the frequency was found, but $G^{\prime \prime}$ showed weak dependency of frequency.

Storage modulus $\left(G^{\prime}\right)$ is a good measure of material stiffness [50], the larger $G^{\prime}$ reflects the larger strength of the material [51, 52]. Figure 4a showed that the incorporation of PVP increased the $G^{\prime}$ much when compared with pure PVA hydrogels. With the increase of polymerization degree of PVA, the values of $G^{\prime}$ increased significantly due to the increasing crosslinking density. The results indicate that hydrogels incorporated with PVP and prepared with larger polymerization degree have larger mechanical strength compared to the pure PVA hydrogels, which is consistent with the results obtained in unconfined compression tests. $G^{\prime \prime}$ increased with the increase of polymerization degree, indicating the increasing of viscosity.

\subsection{Compressive fatigue properties}

The compressive fatigue tests of PVA/PVP hydrogels were carried out with a sinusoidal compressive loading mode from 200 to $800 \mathrm{~N}$ for up to 50, 000 cycles at $5 \mathrm{~Hz}$, and the height of hydrogels changed constantly with the sinusoidal compressive loads. The amount of deformation of different hydrogel formulations during a single loading cycle at the initial, medium and later stage of fatigue tests are summarized in Table 2. It demonstrates that the amount of deformation during a single cycle decreased gradually with loading cycles. Hydrogels prepared with

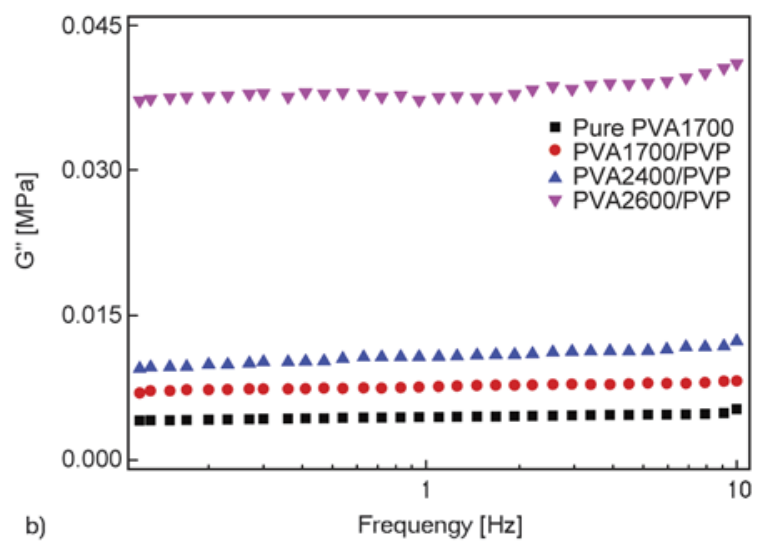

Figure 4. (a) Storage and (b) loss modulus of the hydrogels as function of frequency 
Table 2. The amount of deformation under load during a single loading cycle for PVA/PVP hydrogels at various fatigue test stage

\begin{tabular}{|c|c|c|c|}
\hline Samples & $\begin{array}{c}\boldsymbol{D}_{\mathbf{I}} \\
{[\mathbf{m m}]}\end{array}$ & $\begin{array}{c}\boldsymbol{D}_{\mathbf{M}} \\
{[\mathbf{m m}]}\end{array}$ & $\begin{array}{c}\boldsymbol{D}_{\mathbf{L}} \\
{[\mathbf{m m}]}\end{array}$ \\
\hline PVA1750/PVP & $0.194 \pm 0.007$ & $0.138 \pm 0.004$ & $0.083 \pm 0.003$ \\
\hline PVA2099/PVP & $0.235 \pm 0.011$ & $0.189 \pm 0.009$ & $0.124 \pm 0.012$ \\
\hline PVA2399/PVP & $0.339 \pm 0.019$ & $0.297 \pm 0.015$ & $0.201 \pm 0.009$ \\
\hline
\end{tabular}

${ }^{*} D_{\mathrm{I}}-$ the amount of deformation at the initial stage (1750 cycle), $D_{\mathrm{M}}$ - the amount of deformation at the medium stage

(25000 cycle),

$D_{\mathrm{L}}$ - the amount of deformation at the later stage (50000 cycle).

higher polymerization degree showed larger deformation during the same loading cycle. All the pure PVA hydrogels samples failed after 34000 cycles, so the relevant experimental data were not given here.

The change in normalized deformation of hydrogels under $200 \mathrm{~N}$ with fatigue cycles is given in Figure 5. The deformation increased rapidly at first, and then increased slowly as function of loading cycles, implying a tendence toward the stability. And the final deformation was smaller for hydrogels prepared with higher polymerization degree $(p<0.05)$. The normalized deformation of the three hydrogels after fatigue were $29.3 \pm 1.2,27.0 \pm 0.9$, and $24.9 \pm 0.6 \%$,

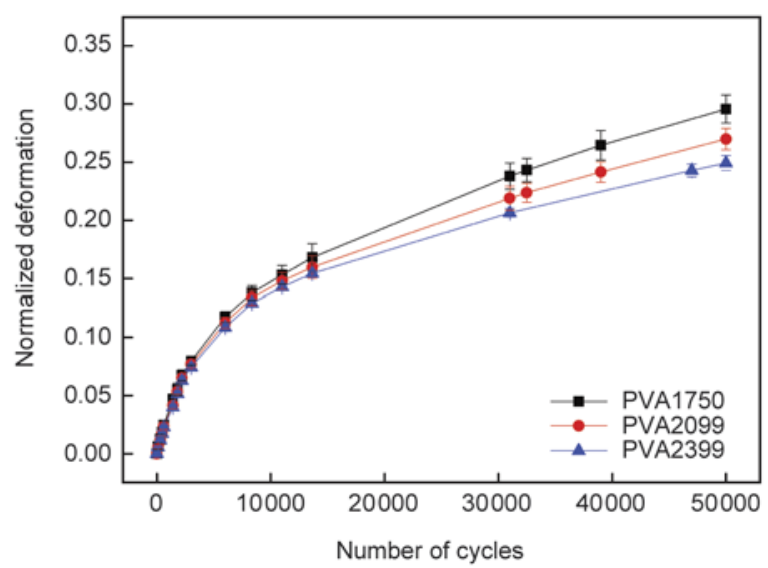

Figure 5. The normalized deformation of various hydrogel formulations as function of fatigue cycles under the load of $200 \mathrm{~N}$

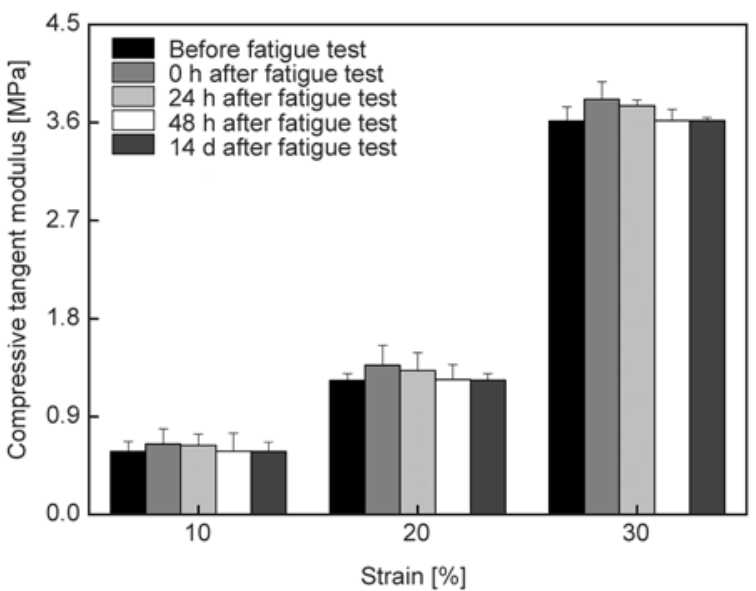

Figure 6. The compressive modulus at 10, 20 and 30\% strain before fatigue and after fatigue test (PVA1750/ PVP)

respectively, with the increase of polymerization degree.

The values of compressive tangent modulus of PVA/PVP hydrogels were calculated at 10, 20 and $30 \%$ strain before fatigue test and $0,24,48 \mathrm{~h}$, and 14 days after test. After subjected to 50000 fatigue cycles, as clearly shown in Figure 6, the tangent modulus of PVA1750/PVP hydrogels showed a significant increase, and then the modulus was recovered to the level before fatigue for $48 \mathrm{~h}$, regardless of the strain level. Table 3 summaries the compressive tangent modulus of the three hydrogel formulations before and $48 \mathrm{~h}$ after fatigue test. The differences between the compressive tangent modulus measured pro-fatigue and post-fatigue were very small for each formulation at each strain level, indicating good recovery capacity for the mechanical property of hydrogels that subjected to 50000 fatigue cycles.

Figure 7 describes the change of normalized height and mass with time for PVA/PVP hydrogels after fatigue tests. All the hydrogel samples that had been subjected to 50000 fatigue cycles showed large deformation and mass loss. And it is obvious that

Table 3. Summary of the compressive modulus of PVA/PVP hydrogels fabricated with various polymerization degree of PVA before fatigue and $48 \mathrm{~h}$ after fatigue tests

\begin{tabular}{|l|c|c|c|}
\hline \multicolumn{1}{|c|}{ Samples } & $\begin{array}{c}\boldsymbol{\varepsilon}_{\mathbf{1 0} \%} \\
{[\mathbf{M P a}]}\end{array}$ & $\begin{array}{c}\boldsymbol{\varepsilon}_{\mathbf{2 0} \%} \\
{[\mathbf{M P a}]}\end{array}$ & $\begin{array}{c}\boldsymbol{\varepsilon}_{\mathbf{3 0}} \\
\text { [MPa] }\end{array}$ \\
\hline PVA1750 (before fatigues) & $0.583 \pm 0.006$ & $1.235 \pm 0.012$ & $3.612 \pm 0.019$ \\
\hline PVA1750 (after fatigues) & $0.588 \pm 0.011$ & $1.242 \pm 0.020$ & $3.618 \pm 0.017$ \\
\hline PVA2099 (before fatigues) & $0.654 \pm 0.004$ & $1.457 \pm 0.007$ & $5.183 \pm 0.013$ \\
\hline PVA2099 (after fatigues) & $0.659 \pm 0.017$ & $1.458 \pm 0.015$ & $5.185 \pm 0.012$ \\
\hline PVA2399 (before fatigues) & $0.752 \pm 0.014$ & $1.982 \pm 0.018$ & $5.813 \pm 0.018$ \\
\hline PVA2399 (after fatigues) & $0.765 \pm 0.016$ & $1.983 \pm 0.021$ & $5.815 \pm 0.020$ \\
\hline
\end{tabular}



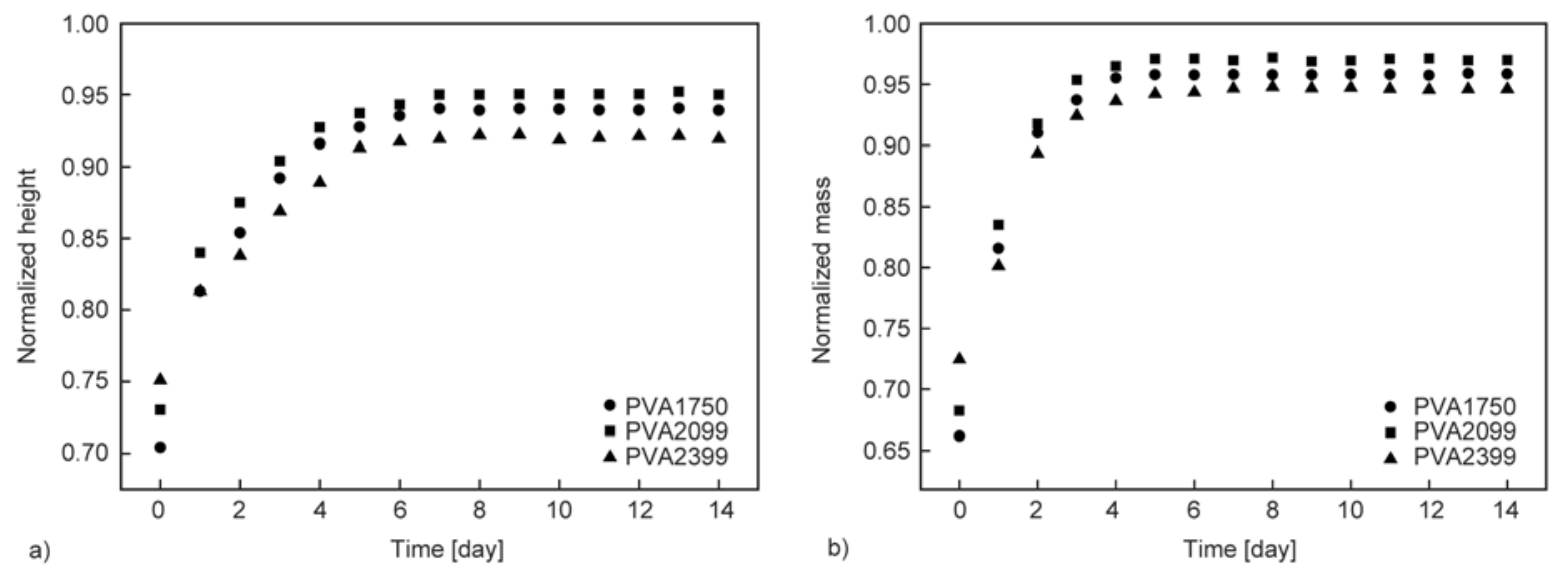

Figure 7. The change of normalized (a) height and (b) mass over time for various PVA/PVP hydrogel formulations after fatigue tests

both height and mass of each hydrogel formulation recovered rapidly within the first 2 days of unrestrained recovery in PBS solution, followed by a slow recovery rate, and then reached a plateau value within one week. It is notable that though both height and mass finally recovered to more than $90 \%$ of their initial values, they could not reach to their original state completely. It is shown from Figure 7 that hydrogels prepared with low polymerization degree experienced more deformation and mass loss during fatigues, but showed better recovery capacity after fatigues than hydrogels fabricated with high polymerization degree.

\subsection{Friction property}

Figure 8 gives the friction coefficient evolution over time during sliding. It is shown that the friction coefficient of PVA/PVP hydrogels was low at the initial stage, and then the friction coefficient increased gradually as time prolonging and tended

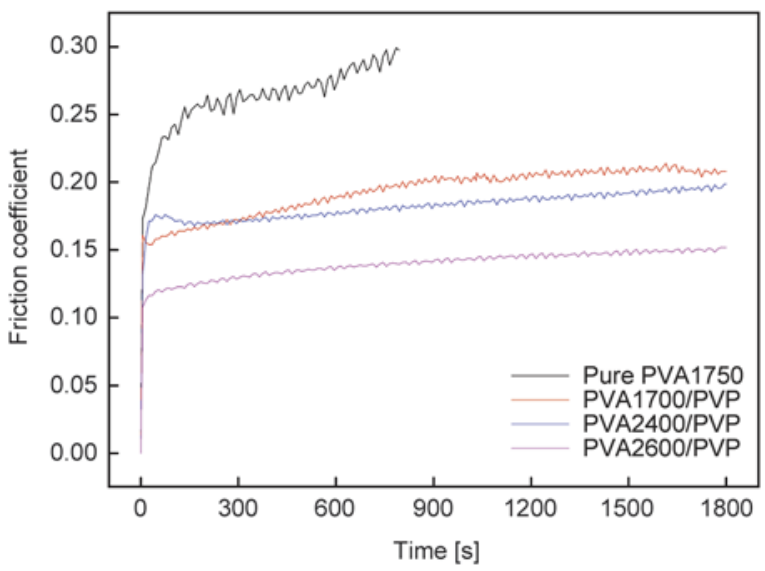

Figure 8. Friction coefficient evolution of pure PVA and PVA/PVP hydrogels during sliding to stable. The three-dimensional porous biphasic structure of hydrogels accounted for this. When the applied normal load was applied to the hydrogel, most of the load was supported by the fluid phase, leading to the relatively low friction coefficient. As the time going, more interstitial water within the porous structure of hydrogels was squeezed out away the contact area, while there was insufficient time for the non-contact area to rehydration. Thus the proportion of load supported by the solid phase was continuously increased, which enlarged the solid-to-solid contact and increased the friction coefficient, and then reached to a relatively stable level $[23,53]$. As shown in Figure 8, the friction coefficient of the pure PVA hydrogel increased rapidly, and the samples were worn out within $800 \mathrm{~s}$. After blended with PVP, the hydrogel was kept relatively low level of friction during the entire friction tests, which was due to the higher hydrophilcity of PVP and the resulting improved lubricity. The friction coefficient of hydrogels decreased with the increasing polymerization degree, as the hydrogel prepared with high polymerization degree showed better load-bearing capacity and then less contact area during sliding.

\section{Discussion}

During daily activities, the synovial joint is routinely subjected to static as well as dynamic loads. Dynamic loading, which causes intra-tissue fluid flow and dynamic tissue deformation, can stimulate the synthesis of matrix molecular at certain amplitudes and frequencies [54], but can also lead to cartilage failure [39]. Researchers always investigated the fatigue behavior of cartilage replacements by 
using the dynamic loading with strain controlled, and the strain was limited to $15 \%$. In general, $15 \%$ strain corresponds to a very small load for hydrogels, and this load would far less than the physiological load that the cartilage is subjected, which is of several times body weight. $15 \%$ strain is very close to the linear elastic region of hydrogels, so it is expected that the hydrogel would not be failure for even a very long term loading cycles. In addition, the reports about the recovery behavior of cartilage prosthesis after fatigue test under large load are few. So in the present study, we were interested in characterizing the compressive fatigue behavior of PVA/ PVP hydrogels as cartilage prosthesis under dynamic loading from 200 to $800 \mathrm{~N}$ for up to 50000 cycles at $5 \mathrm{~Hz}$ and their recovery behavior after fatigue failure.

PVA/PVP hydrogels show the creep phenomenon under static loading for a period of time, called the static creep here. The consecutive sinusoidal loading for up to 50000 cycles at $5 \mathrm{~Hz}$ could have produced creep effects as well [55], defined as the dynamic creep. The same tendency of deformation varying with time (or the cycles) was found in both static and dynamic creep (Figure 3 and 6). The rapid deformation at first is attributed to the fast effusion of interstitial water from the loading zone. The extrusion rate of the water decreased with time, and the slow motion of molecular chains was dragged by large internal friction [56], resulting in the decreased deformation rate. The deformation would reach to a stable level eventually. This deformation tendency with time under load is similar to the natural articular cartilage [57]. The deformation of the natural cartilage tissue could achieve a cyclic steady state faster than under a static load $[58,59]$. However, there seems to be a contradiction between the experimental results and the expectation in the present study. That is primarily because the differences between the local deformation resulting from the indentation creep with a spherical indenter and the block deformation caused by the compression plate. The creep rate of cartilage was dependent on its permeability [60], and its equilibrium time of creep was demonstrated to be approximately $60 \mathrm{~min}$ [57]. While the deformation of both static and dynamic creep for PVA/PVP hydrogels was increasing during the entire testing time, instead of reaching an equilibrium value. The thicker sample delays the equilibrium time of creep may account for this [61].
Considering the movement of the interstitial water during the sinusoidal loading can lead to an explanation of the recovery behavior during fatigue. Figure 9 provides a visual picture for the microstructural change of a PVA/PVP hydrogel sample during a single sinusoidal loading cycle. During loading phase, the polymer network deforms, and the interstitial water migrated from the loaded region to the unloaded region due to the pressure gradient caused by the differences between the applied stress and the hydrogel swelling pressure (Figure $9 \mathrm{~b}$ to $9 \mathrm{c}$ ). While during the uploading phase, the flow direction of interstitial water changed to the reverse direction, resulting in the re-swelling of the hydrogel; but the flow was driven under the action of a much lower pressure gradient, because the swelling pressure, which would govern the recovery rate, was far lower than the stress resulting from the loads acting. So it was impossible for the hydrogel sample to fully recover during such a very short period of time $(0.1 \mathrm{~s})$, that is, $h^{\prime}{ }_{200}$ was less than $h_{200}$. This result is consistent with Maroudas's predictions [62]. Barker and Seedhom [63] has demonstrated that the contact between the flat-ended indenter and the natural cartilage, even under a small tare load of about 0.014 MPa, has almost blocked the path of the fluid being imbibed by the cartilage matrix during the recovery phase of the loading cycle. In the present experiment, the large normal load was maintained on the hydrogel surface throughout the test, which significantly decreased the permeability of the hydrogel surface. As a result, the fluid path was further restricted, and the recovery was greatly restrained during the loading cycle.

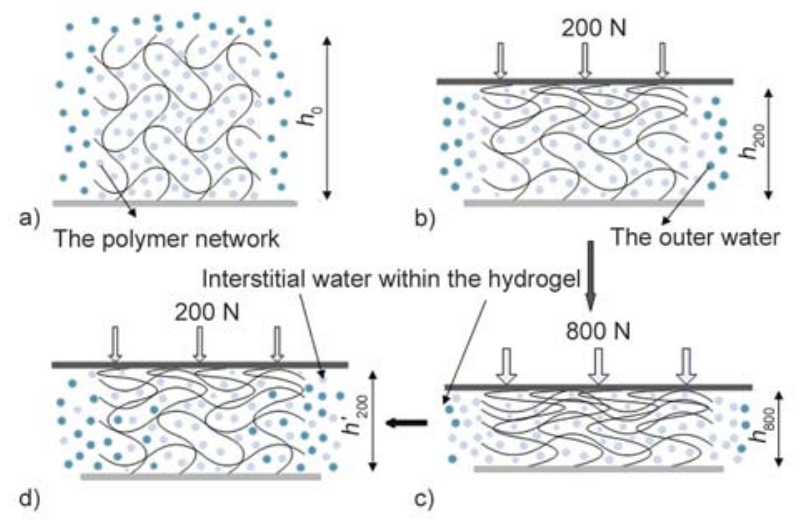

Figure 9. The diagrams of microstructure of PVA/PVP hydrogel during a single loading cycle. (a) represents the unloaded hydrogel. (b), (c) and (d) denote the state of hydrogels loaded from 200 to $800 \mathrm{~N}$, and then unloaded to $200 \mathrm{~N}$, respectively. 
After unrestrained recovery in fresh PBS solution after fatigue test, there was no change in compressive tangent modulus of samples before and after fatigue tests for $48 \mathrm{~h}$ after fatigue tests, indicating intact and structurally unchanged for the hydrogel samples. Height and mass of hydrogels presented greater recovery at the first 2 days, after that the recovery of all hydrogel formulations reached a plateau value gradually. Both height and mass finally recovered to more than $90 \%$ of their initial values. That is the permanent deformation, due to the creep effect caused by the cumulative fatigue, prevented the complete recovery of the tested hydrogel. In the present experiment it was observed that hydrogel prepared with lower polymerization degree of PVA showed faster recovery rate and less permanent deformation than the one prepared with higher polymerization degree. Under the same loading condition, hydrogels with lower polymerization degree is subjected to larger strains, and the swelling pressure during the recovery phase after fatigue test must be higher than that of hydrogels with higher polymerization degree. The less porosity and the lower permeability resulting from the denser network structure also account for the slower recovery of hydrogel with higher polymerization degree. Hydrogel prepared with higher polymerization degree shows larger permanent deformation, it is attributed to the excessive viscous losses (Figure 4b).

In the present study, PVA/PVP hydrogel sustains dynamic compressive load from 200 to $800 \mathrm{~N}$ for up to 50000 cycles with structurally unchanged, and shows good recovery capacity after fatigue test, indicating its potential to be used as articular cartilage prosthesis. More detailed studies are still needed in the following researches, including longer fatigue cycles, multiple fatigue recovery. Combining the micro-properties with the bulk mechanical properties via techniques such as nanoindentation to explore the fatigue failure mechanism of hydrogels in depth is also another important research direction. The compressive fatigue properties of the natural articular cartilage would be evaluated and then make comparisons with that of replacement materials for better material design.

\section{Conclusions}

In this study, PVA/PVP hydrogel were synthesized by a repeated freezing-thawing method. The incorpo- ration of PVP into PVA hydrogel stabilized its mechanical property and improves the lubricity. Higher compressive modulus was observed for hydrogel prepared with higher polymerization degree, and the friction coefficient decreased with the increase of polymerization degree. In order to explore the compressive fatigue properties of PVA/PVP hydrogels as cartilage prosthesis, the hydrogel samples were subjected to cyclic compressive loading from 200 to $800 \mathrm{~N}$ at $5 \mathrm{~Hz}$ for up to 50000 cycles, and their recovery behavior after fatigue test was evaluated. The result showed that the height of the hydrogel rapidly decreased at first and gradually became stable with loading cycles. After unrestrained recovery in fresh PBS solution after fatigue test, the compressive tangent modulus recovered to its original level within $48 \mathrm{~h}$, but the geometry of samples could not regain their original state completely due to the creep effects. Hydrogels prepared with lower polymerization degree of PVA showed better recovery capacity from cyclic loading.

\section{Acknowledgements}

This project is supported by the National Natural Science Foundation of China (Grant nos. 11172142 and 51575278), and the Priority Academic Program Development of Jiangsu Higher Education Institutions (PAPD).

\section{References}

[1] Schulz R. M., Bader A.: Cartilage tissue engineering and bioreactor systems for the cultivation and stimulation of chondrocytes. European Biophysics Journal, 36, 539-568 (2007).

DOI: $10.1007 / \mathrm{s} 00249-007-0139-1$

[2] Rawal B. R., Ribeiro R., Chouksey M., Tripathi K.: Biomaterials for cartilage repair: A review. Journal of Medical Sciences, 13, 615-620 (2013). DOI: $10.3923 / \mathrm{jms} .2013 .615 .620$

[3] Peretti G. M., Pozzi A., Ballis R., Deponti D., Pellacci F.: Current surgical options for articular cartilage repair. Advances in Minimally Invasive Surgery and Therapy for Spine and Nerves, 108, 213-219 (2011). DOI: $10.1007 / 978-3-211-99370-533$

[4] Hoffman A. S.: Hydrogels for biomedical applications. Advanced Drug Delivery Reviews, 54, 3-12 (2002). DOI: $10.1016 / \mathrm{S} 0169-409 \mathrm{X}(01) 00239-3$

[5] Peppas N. A., Hilt J. Z., Khademhosseini A., Langer R.: Hydrogels in biology and medicine: From molecular principles to bionanotechnology. Advanced Materials, 18, 1345-1360 (2006).

DOI: $10.1002 / \mathrm{adma} .200501612$ 
[6] Gonzalez J. S., Alvarez V. A.: Mechanical properties of polyvinylalcohol/hydroxyapatite cryogel as potential artificial cartilage. Journal of the Mechanical Behavior of Biomedical Materials, 34, 47-56 (2014). DOI: 10.1016/j.jmbbm.2014.01.019

[7] Spiller K. L., Maher S. A., Lowman A. M.: Hydrogels for the repair of articular cartilage defects. Tissue Engineering Part B, 17, 281-299 (2011).

DOI: $10.1089 /$ ten.teb.2011.0077

[8] Toh W. S., Lee E. H., Guo X-M., Chan J. K., Yeow C. H., Choo A. B., Cao T.: Cartilage repair using hyaluronan hydrogel-encapsulated human embryonic stem cellderived chondrogenic cells. Biomaterials, 31, 69686980 (2010).

DOI: 10.1016/j.biomaterials.2010.05.064

[9] Lee C. R., Grodzinsky A. J., Spector M.: The effects of cross-linking of collagen-glycosaminoglycan scaffolds on compressive stiffness, chondrocyte-mediated contraction, proliferation and biosynthesis. Biomaterials, 22, 3145-3154 (2001).

DOI: $10.1016 / \mathrm{S} 0142-9612(01) 00067-9$

[10] Hao T., Wen N., Cao J-K., Wang H-B., Lü S-H., Liu T., Lin Q-X., Duan C-M., Wang C-Y.: The support of matrix accumulation and the promotion of sheep articular cartilage defects repair in vivo by chitosan hydrogels. Osteoarthritis and Cartilage, 18, 257-265 (2010). DOI: $10.1016 /$ j.joca.2009.08.007

[11] Ho S. T., Cool S. M., Hui J. H., Hutmacher D. W.: The influence of fibrin based hydrogels on the chondrogenic differentiation of human bone marrow stromal cells. Biomaterials, 31, 38-47 (2010).

DOI: 10.1016/j.biomaterials.2009.09.021

[12] Bray J. C., Merrill E. W.: Poly(vinyl alcohol) hydrogels for synthetic articular cartilage material. Journal of Biomedical Materials Research, 7, 431-443 (1973). DOI: $10.1002 / \mathrm{jbm} .820070506$

[13] Hassan C. M., Peppas N. A.: Structure and applications of poly(vinyl alcohol) hydrogels produced by conventional crosslinking or by freezing/thawing methods. Advances in Polymer Science, 153, 37-65 (2000). DOI: 10.1007/3-540-46414-X_2

[14] Bryant S. J., Arthur J. A., Anseth K. S.: Incorporation of tissue-specific molecules alters chondrocyte metabolism and gene expression in photocrosslinked hydrogels. Acta Biomaterialia, 1, 243-252 (2005).

DOI: $10.1016 /$ j.actbio.2004.11.003

[15] Cho J. H., Kim S-H., Park K. D., Jung M. C., Yang W. I., Han S. W., Noh J. Y., Lee J. W.: Chondrogenic differentiation of human mesenchymal stem cells using a thermosensitive poly( $N$-isopropylacrylamide) and watersoluble chitosan copolymer. Biomaterials, 25, 57435751 (2004).

DOI: $10.1016 /$ j.biomaterials.2004.01.051

[16] Gong J. P., Katsuyama Y., Kurokawa T., Osada Y.: Double-network hydrogels with extremely high mechanical strength. Advanced Materials, 15, 1155-1158 (2003). DOI: $10.1002 / \mathrm{adma} .200304907$
[17] Stammen J. A., Williams S., Ku D. N., Guldberg R. E.: Mechanical properties of a novel PVA hydrogel in shear and unconfined compression. Biomaterials, 22, 799-806 (2001). DOI: $10.1016 / \mathrm{S} 0142-9612(00) 00242-8$

[18] Baker M. I., Walsh S. P., Schwartz Z., Boyan B. D.: A review of polyvinyl alcohol and its uses in cartilage and orthopedic applications. Journal of Biomedical Materials Research Part B: Applied Biomaterials, 100, 14511457 (2012). DOI: $10.1002 / \mathrm{jbm} . \mathrm{b} .32694$

[19] Pan Y-S., Xiong D-S., Ma R-Y.: Preparation and swelling behavior of polyvinyl alcohol physiological saline gel. Journal of Central South University of Technology, 13, 27-31 (2006). DOI: $10.1007 / \mathrm{s} 11771-006-0101-\mathrm{x}$

[20] Xie L., Jiang M., Dong X., Bai X., Tong J., Zhou J.: Controlled mechanical and swelling properties of poly (vinyl alcohol)/sodium alginate blend hydrogels prepared by freeze-thaw followed by $\mathrm{Ca}^{2+}$ crosslinking. Journal of Applied Polymer Science, 124, 823-831 (2012). DOI: $10.1002 / a p p .35083$

[21] Ma R., Xiong D., Miao F., Zhang J., Peng Y.: Novel PVP/PVA hydrogels for articular cartilage replacement. Materials Science and Engineering: C, 29, 1979-1983 (2009). DOI: $10.1016 /$ j.msec.2009.03.010

[22] Zhang L., Wang Z. P., Xu C., Li Y., Gao J. P., Wang W., Liu Y.: High strength graphene oxide/polyvinyl alcohol composite hydrogels. Journal of Materials Chemistry, 21, 10399-10406 (2011). DOI: 10.1039/C0JM04043F

[23] Shi Y., Xiong D.: Microstructure and friction properties of PVA/PVP hydrogels for articular cartilage repair as function of polymerization degree and polymer concentration. Wear, 305, 280-285 (2013).

DOI: $10.1016 /$ j.wear.2012.12.020

[24] Dunn A. C., Sawyer W. G., Angelini T. E.: Gemini interfaces in aqueous lubrication with hydrogels. Tribology Letters, 54, 59-66 (2014).

DOI: $10.1007 / \mathrm{s} 11249-014-0308-1$

[25] Chen K., Zhang D., Wang S.: Start-up friction properties of poly(vinyl alcohol)/nano-hydroxyapatite/silk composite hydrogel. Materials Express, 3, 265-272 (2013).

DOI: $10.1166 / \operatorname{mex} .2013 .1127$

[26] Tadavarthy S. M., Moller J. H., Amplatz K.: Polyvinyl alcohol (Ivalon) - A new embolic material. The American Journal of Roentgenology, Radium Therapy, and Nuclear Medicine, 125, 609-616 (1975).

[27] Mallapragada S. K., Peppas N. A.: Dissolution mechanism of semicrystalline poly(vinyl alcohol) in water. Journal of Polymer Science Part B: Polymer Physics, 34, 1339-1346 (1996).

DOI: $10.1002 /($ SICI)1099-0488(199605)34:7<1339:: AID-POLB15>3.0.CO;2-B 
[28] Maolin Z., Hongfei H., Yoshii F., Makuuchi K.: Effect of kappa-carrageenan on the properties of poly $(N$-vinyl pyrrolidone)/kappa-carrageenan blend hydrogel synthesized by $\gamma$-radiation technology. Radiation Physics and Chemistry, 57, 459-464 (2000).

DOI: 10.1016/S0969-806X(99)00415-6

[29] Wagner D. H.: Nanocomposites: Paving the way to stronger materials. Nature Nanotechnology, 2, 742-744 (2007).

DOI: $10.1038 /$ nnano.2007.401

[30] Abd El-Mohdy H. L., Ghanem S.: Biodegradability, antimicrobial activity and properties of PVA/PVP hydrogels prepared by $\gamma$-irradiation. Journal of Polymer Research, 16, 1-10 (2009).

DOI: $10.1007 / \mathrm{s} 10965-008-9196-0$

[31] Leone G., Consumi M., Greco G., Bonechi C., Lamponi S., Rossi C., Magnani A.: A PVA/PVP hydrogel for human lens substitution: Synthesis, rheological characterization, and in vitro biocompatibility. Journal of Biomedical Materials Research Part B: Applied Biomaterials, 97, 278-288 (2011).

DOI: $10.1002 / \mathrm{jbm} \cdot \mathrm{b} .31813$

[32] Thomas J., Lowman A., Marcolongo M.: Novel associated hydrogels for nucleus pulposus replacement. Journal of Biomedical Materials Research Part A, 67, 1329-1337 (2003).

DOI: $10.1002 / \mathrm{jbm} . \mathrm{a} .10119$

[33] Bellucci G., Seedhom B. B.: Mechanical behaviour of articular cartilage under tensile cyclic load. Rheumatology, 40, 1337-1345 (2001).

DOI: $10.1093 /$ rheumatology/40.12.1337

[34] Sakai N., Hagihara Y., Furusawa T., Hosoda N., Sawae Y., Murakami T.: Analysis of biphasic lubrication of articular cartilage loaded by cylindrical indenter. Tribology International, 46, 225-236 (2012).

DOI: 10.1016/j.triboint.2011.03.016

[35] Mauck R. L., Seyhan S. L., Ateshian G. A., Hung C. T.: Influence of seeding density and dynamic deformational loading on the developing structure/function relationships of chondrocyte-seeded agarose hydrogels. Annals of Biomedical Engineering, 30, 10461056 (2002).

DOI: $10.1114 / 1.1512676$

[36] Bryant S. J., Chowdhury T. T., Lee D. A., Bader D. L., Anseth K. S.: Crosslinking density influences chondrocyte metabolism in dynamically loaded photocrosslinked poly(ethylene glycol) hydrogels. Annals of Biomedical Engineering, 32, 407-417 (2004). DOI: 10.1023/B:ABME.0000017535.00602.ca

[37] Huang A. H., Farrell M. J., Kim M., Mauck R. L.: Longterm dynamic loading improves the mechanical properties of chondrogenic mesenchymal stem cell-laden hydrogels. European Cells and Materials, 19, 72-85 (2010).
[38] Mauck R. L., Soltz M. A., Wang C. B., Wong D. D., Chao P-H. G., Valhmu W. B., Hung C. T., Ateshian G. A.: Functional tissue engineering of articular cartilage through dynamic loading of chondrocyte-seeded agarose gels. Journal of Biomechanical Engineering, 122, 252260 (2000).

DOI: $10.1115 / 1.429656$

[39] Weightman B. O., Freeman M. A. R., Swanson S. A. V.: Fatigue of articular cartilage. Nature, 244, 303-304 (1973). DOI: $10.1038 / 244303 \mathrm{a} 0$

[40] Weightman B.: Tensile fatigue of human articular cartilage. Journal of Biomechanics, 9, 193-200 (1976). DOI: 10.1016/0021-9290(76)90004-X

[41] Vikingsson L., Gómez-Tejedor J. A., Ferrer G. G., Gómez Ribelles J. L.: An experimental fatigue study of a porous scaffold for the regeneration of articular cartilage. Journal of Biomechanics, 48, 1310-1317 (2015). DOI: 10.1016/j.jbiomech.2015.02.013

[42] Vikingsson L., Ferrer G. G., Gómez-Tejedor J. A., Ribelles J. L.: An 'in vitro' experimental model to predict the mechanical behavior of macroporous scaffolds implanted in articular cartilage. Journal of the Mechanical Behavior of Biomedical Materials, 32, 125-131 (2014).

DOI: $10.1016 /$ j.jmbbm.2013.12.024

[43] Peppas N. A., Merrill E. W.: Differential scanning calorimetry of crystallized PVA hydrogels. Journal of Applied Polymer Science, 20, 1457-1465 (1976). DOI: 10.1002/app.1976.070200604

[44] Pan Y., Xiong D.: Study on compressive mechanical properties of nanohydroxyapatite reinforced poly(vinyl alcohol) gel composites as biomaterial. Journal of Materials Science: Materials in Medicine, 20, 1291-1297 (2009).

DOI: $10.1007 / \mathrm{s} 10856-008-3679-8$

[45] Clark J. M.: Variation of collagen fiber alignment in a joint surface: A scanning electron microscope study of the tibial plateau in dog, rabbit, and man. Journal of Orthopaedic Research, 9, 246-257 (1991).

DOI: $10.1002 /$ jor.1100090213

[46] Holloway J. L., Lowman A. M., Palmese G. R.: The role of crystallization and phase separation in the formation of physically cross-linked PVA hydrogels. Soft Matter, 9, 826-833 (2013).

DOI: $10.1039 / \mathrm{c} 2 \mathrm{sm} 26763 \mathrm{~b}$

[47] McNary S. M., Athanasiou K. A., Reddi A. H.: Engineering lubrication in articular cartilage. Tissue Engineering Part B: Reviews, 18, 88-100 (2012). DOI: $10.1089 /$ ten.teb.2011.0394

[48] Calvet D., Wong J. Y., Giasson S.: Rheological monitoring of polyacrylamide gelation: Importance of crosslink density and temperature. Macromolecules, 37, 7762-7771 (2004).

DOI: $10.1021 / \mathrm{ma} 049072 \mathrm{r}$ 
[49] Michailova V., Titeva S., Kotsilkova R., Krusteva E., Minkov E.: Influence of aqueous medium on viscoelastic properties of carboxymethylcellulose sodium, hydroxypropyimethyl cellulose, and thermally pregelatinized starch gels. Colloids and Surfaces A: Physicochemical and Engineering Aspects, 149, 515-520 (1999).

DOI: 10.1016/S0927-7757(98)00608-6

[50] Ricciardi R., Gaillet C., Ducouret G., Lafuma F., Lauprêtre F.: Investigation of the relationships between the chain organization and rheological properties of atactic poly(vinyl alcohol) hydrogels. Polymer, 44, 33753380 (2003).

DOI: $10.1016 / \mathrm{S} 0032-3861(03) 00246-5$

[51] Sosnik A., Sefton M. V.: Semi-synthetic collagen/poloxamine matrices for tissue engineering. Biomaterials, 26, 7425-7435 (2005).

DOI: 10.1016/j.biomaterials.2005.05.086

[52] Haines L. A., Rajagopal K., Ozbas B., Salick D. A., Pochan D. J., Schneider J. P.: Light-activated hydrogel formation via the triggered folding and self-assembly of a designed peptide. Journal of the American Chemical Society, 127, 17025-17029 (2005). DOI: $10.1021 / \mathrm{ja} 0547190$

[53] Katta J., Jin Z., Ingham E., Fisher J.: Effect of nominal stress on the long term friction, deformation and wear of native and glycosaminoglycan deficient articular cartilage. Osteoarthritis and Cartilage, 17, 662-668 (2009). DOI: 10.1016/j.joca.2008.10.008

[54] Davisson T., Kunig S., Chen A., Sah R., Ratcliffe A.: Static and dynamic compression modulate matrix metabolism in tissue engineered cartilage. Journal of Orthopaedic Research, 20, 842-848 (2002). DOI: $10.1016 / \mathrm{S} 0736-0266(01) 00160-7$
[55] Joshi A., Fussell G., Thomas J., Hsuan A., Lowman A., Karduna A., Vresilovic E., Marcolongo M.: Functional compressive mechanics of a PVA/PVP nucleus pulposus replacement. Biomaterials, 27, 176-184 (2006). DOI: 10.1016/j.biomaterials.2005.06.003

[56] Reynaud B., Quinn T. M.: Tensorial electrokinetics in articular cartilage. Biophysical Journal, 91, 23492355 (2006).

DOI: 10.1529/biophysj.106.082263

[57] Meng W-C., Dong Q-R.: The experiment study on the compression properties of articular cartilage. Journal of Medical Biomechanics, 18, 28-33 (2003).

[58] Linn F. C.: Lubrication of animal joints. I. The arthrotripsometer. Journal of Bone and Joint Surgery, 49, 10791098 (1967).

[59] Simon W. H.: Scale effects in animal joints. I. Articular cartilage thickness and compressive stress. Arthritis and Rheumatism, 13, 244-255 (1970).

DOI: $10.1002 / \operatorname{art} .1780130305$

[60] Li L. P., Korhonen R. K., Iivarinen J., Jurvelin J. S., Herzog W.: Fluid pressure driven fibril reinforcement in creep and relaxation tests of articular cartilage. Medical Engineering and Physics, 30, 182-189 (2008). DOI: $10.1016 / \mathrm{j}$. medengphy.2007.03.001

[61] Mow V. C., Holmes M. H., Lai W. M.: Fluid transport and mechanical properties of articular cartilage: A review. Journal of Biomechanics, 17, 377-394 (1984). DOI: 10.1016/0021-9290(84)90031-9

[62] Maroudas A.: Physico-chemical properties of articular cartilage. Pitman Medical, London (1973).

[63] Barker M. K., Seedhom B. B.: The relationship of the compressive modulus of articular cartilage with its deformation response to cyclic loading: Does cartilage optimize its modulus so as to minimize the strains arising in it due to the prevalent loading regime? Rheumatology, 40, 274-284 (2001).

DOI: $10.1093 /$ rheumatology/40.3.274 